\title{
A Computation of a Crystalline Flow Starting from Non-admissible Polygon Using Expanding Selfsimilar Solutions
}

\author{
Hidekata Hontani ${ }^{1}$, Mi-Ho Giga ${ }^{2}$, Yoshikazu Giga ${ }^{2}$, and Koichiro Deguchi ${ }^{3}$ \\ 1 Department of Informatics, Yamagata University \\ 4-3-16, Yonezawa, Yamagata, 992-8510 Japan \\ hontani@yz . yamagata-u.ac.jp \\ 2 Department of Mathematics, Hokkaido University \\ nishi-8, kita-10-jo, Sapporo, Hokkaido, 060-0810 Japan \\ \{mihogiga, giga\}@math.sci.hokudai.ac.jp \\ 3 Department of System Information Science, Tohoku University \\ Aramaki-aza Aoba01, Aoba-ku, Sendai, Miyagi, 980-8579, Japan \\ kodeg@fractal.is.tohoku.ac.jp
}

\begin{abstract}
A numerical method for obtaining a crystalline flow from a given polygon is presented. A crystalline flow is a discrete version of a classical curvature flow. In a crystalline flow, a given polygon evolves, and it remains polygonal through the evolving process. Each facet moves keeping its normal direction, and the normal velocity is determined by the length of the facet. In some cases, a set of new facets sprout out at the very beginning of the evolving process. The facet length is governed by a system of singular ordinary differential equations. The proposed method solves the system of ODEs, and obtain the length of each new facet, systematically. Experimental results show that the method obtains a crystalline flow from a given polygon successfully.
\end{abstract}

\section{Introduction}

An evolution based multi-scale analysis plays an important role to characterize a contour figure in an image 1] 2]. A family of evolving contours that is called a curvature flow is used for this analysis. In the flow, every point in the contour moves toward the normal direction of the contour with the velocity $V$ determined by the curvature $\kappa$ at each position. As a given contour evolves, its shape changes. Observing the change, those methods extract shape features of a given contour. The flow given by setting $V=\kappa$ is called the curve shortening flow, which is widely used for the multi-scale analysis [3] [4] [5] 6].

For the computation of the curve shortening flow, there have been proposed several methods. For those methods, the representation of a contour figure is quite important, since each of those methods represents the smoothly evolving curve discretely. For example, the Gaussian-based method 3 represents an evolving contour figure by a set of points that are equally spaced in the contour. The coordinates of the $i$ th point is represented as $(x(i \Delta), y(i \Delta))$ where $\Delta$ denotes 
the interval between adjacent points. The method iterates two processes: (1) smoothing both $x(\cdot)$ and $y(\cdot)$ with a small scale Gaussian filter, and (2) resampling the resulted contour at equal intervals after the smoothing. The resampling process is needed because the arc length changes as the contour evolves. This method can obtain the curvature flow without computing the curvature. On the other hand, the resampling process deforms the shape of the represented contour figure slightly at each iteration step. In addition, the interval $\Delta$ changes at each iteration because $\Delta$ must aliquot of the total peripheral length, but it is not realizable.

A level set method[7][8][9] is a powerful tool for obtaining a curvature flow. It is widely used. The method represents an evolving interface as the zero level set of a higher dimensional function $\phi$. For example, an evolving contour in a $x-y$ plane is represented as the zero level set of the evolving function $\phi(x, y ; t)$. This method needs no arc length parameter along the contour. For obtaining the curvature flow, we only need to solve the level set equation $\phi_{t}+\kappa|\nabla \phi|=0$. In the computation, the function $\phi$ is discretely represented on fixed pixels, and finite difference operators are used for computing the spatial derivatives. The operators' width is usually two or three pixels. If there is a small part in the evolving contour that is comparable to the operators' width, then, the computed values do not approximate well the spatial derivatives.

In [10] and [11, a crystalline flow is proposed. The crystalline flow is a special family of evolving polygons. It can be regarded as a discrete version of a classical curvature flow. A given contour figure in an image can be often interpreted as a polygon. In the evolving process of the crystalline flow, a given polygon remains polygonal through the evolving process. Each facet moves keeping its normal direction. The velocity is determined by the non-local curvature, which depends on the length of the facet. Polygons are well represented in a discrete manner. Different from a classical curvature flow, it is not difficult to compute the nonlocal curvature correctly, and to obtain the crystalline flow if an appropriate initial polygon is given. The crystalline flow proposed in [10] and [11] restricts initial polygonal contour.

In [12] and [13, a level set formulation was extended to handle some family of non-local curvature flow including a crystalline flow. Any polygon can be given as an initial contour of a crystalline flow by a level set formulation proposed in 12 and 13 . In some cases, new facets sprout out at corners of a given polygon, at the very beginning of the evolving process. Once new facets sprout out, then, no new facet sprout out any more, and the number of facets decreases monotonically, as time increases. Recently, a system of singular ordinary differential equations of facet length has been studied to handle new facets sprouting out 14 . In this article, we present a numerical method for solving the system of ODEs and for obtaining a crystalline flow from a given polygon. The length of each new facet is systematically calculated. The proposed method enables to use any simple and convex polygon as the Wulff shape, which determines the nonlocal curvature of each facet. 


\section{Crystalline Flow}

\subsection{Weighted Curvature Flow}

First, we recall the notion of the weighted curvature. Let $\gamma$ be a continuous, convex function on $\boldsymbol{R}^{2}$ which is positively homogeneous of degree one, i.e., $\gamma(\lambda \boldsymbol{p})=\lambda \gamma(\boldsymbol{p})$ for all $\boldsymbol{p} \in \boldsymbol{R}^{2}, \lambda>0$. Assume that $\gamma(\boldsymbol{p})>0$ for $\boldsymbol{p} \neq 0$. For a moment assume that $\gamma$ is smooth (except the origin). For an oriented curve $S$ with the orientation $\boldsymbol{n}$, which is a unit normal, we call $\Lambda_{\gamma}(\boldsymbol{n})=-\operatorname{div}(\boldsymbol{\xi}(\boldsymbol{n}))$ the weighted curvature of $S$ in the direction of $\boldsymbol{n}$, where $\boldsymbol{\xi}=\nabla \gamma$. We note that the weighted curvature of $S$ is the first variation of $I(S)$ with respect to a variation of the area enclosed by $S$; here $I(S)$ is defined by

$$
I(S)=\int_{S} \gamma(\boldsymbol{n}) d s,
$$

where $d s$ denotes the line element; $I(S)$ is called the interfacial energy with an interfacial energy density $\gamma$. We recall that the Wulff shape defined by

$$
W_{\gamma}=\underset{|\boldsymbol{m}|=1}{\bigcap}\left\{\boldsymbol{x} \in \boldsymbol{R}^{2} ; \boldsymbol{x} \cdot \boldsymbol{m} \leq \gamma(\boldsymbol{m})\right\}
$$

is the unique minimizer of $I(S)$ among all $S$ whose enclosed area is the same as $W_{\gamma}$ (see e.g. [15). If $\gamma(\boldsymbol{p})=|\boldsymbol{p}|$, then $\Lambda_{\gamma}$ is the usual curvature, and $W_{\gamma}$ is nothing but a unit disk. For any $\gamma$ the weighted curvature of $\partial W_{\gamma}$ always equals -1 , so $W_{\gamma}$ plays the role of a unit disk for the usual curvature.

We consider a motion of an evolving curve $\Gamma_{t}$ governed by the anisotropic curvature flow equation of the form

$$
V=\Lambda_{\gamma}(\boldsymbol{n})
$$

on $\Gamma_{t}$, where $V$ denotes the normal velocity of $\left\{\Gamma_{t}\right\}$ in the direction of $\boldsymbol{n}$. When $\gamma(\boldsymbol{p})=|\boldsymbol{p}|$, equation (2) becomes the curve shortening equation.

There are several methods to track evolution of $\Gamma_{t}$; one of a typical method is the level-set method (see [7, 8], [9], [16]). If $\gamma$ is $C^{2}$ except the origin, global unique solvability for (2) is established by [9] (see also [17). However, when $\gamma$ has corners, conventional notion of a solution including viscosity solutions does not apply to (2).

If Frank diagram of $\gamma$ :

$$
\text { Frank } \gamma=\left\{\boldsymbol{p} \in \mathbf{R}^{2} ; \gamma(\boldsymbol{p}) \leq 1\right\}
$$

is a convex polygon, $\gamma$ is called a crystalline energy (density), and a notion of solution for (2) is proposed by [10] and [11] independently by restricting $\left\{\Gamma_{t}\right\}$ as a special family of evolving polygonal curves called admissible. Even for more general $\gamma$ with corners not necessarily crystalline energy, the level-set approach for (2) and more general equations is successfully extended by [13] (see also [12]), although the problem has nonlocal nature. They introduced a new notion of solution consistent with that in 10] and [11, and proved the global unique solvability at least for a general initial simple curve (not necessarily admissible). 


\subsection{Crystalline Flow}

Here and hereafter we assume that $\gamma$ is a crystalline energy, i.e., Frank $\gamma$ is a convex $M$-polygon. In this section we introduce an evolving polygonal curve called a crystalline flow governed by (2). To track such an evolving polygon, we shall derive a system of ordinary differential equations (ODEs) for the length of sides (facets) of the polygon. For this purpose we need to prepare several notions.

Let $\boldsymbol{q}_{i}(i=1, \ldots, M)$ be vertices of Frank $\gamma$. We call a simple polygonal curve $S$ as an essentially admissible crystal if the outward unit normal vector $\boldsymbol{m}$ and $\hat{\boldsymbol{m}}$ of any adjacent segments (facets) of $S$ satisfy

$$
\frac{(1-\lambda) \boldsymbol{m}+\lambda \hat{\boldsymbol{m}}}{|(1-\lambda) \boldsymbol{m}+\lambda \hat{\boldsymbol{m}}|} \notin \mathcal{N}
$$

for any $\lambda \in(0,1)$, where $\mathcal{N}=\left\{\boldsymbol{q}_{i} /\left|\boldsymbol{q}_{i}\right| ; i=1, \ldots, M\right\}$. Let $J$ be a time interval. We say that a family of polygon $\{S(t)\}_{t \in J}$ is an essentially admissible evolving crystal if $S(t)$ is an essentially admissible crystal for all $t \in J$ and each corner moves continuously differentiably in time. These conditions imply that the orientation of each facet is preserved in $J$. By definition $S(t)$ is of the form $S(t)=\cup_{j=1}^{r} S_{j}(t)$ where $S_{j}(t)$ is a maximal, nontrivial, closed segment and its orientation is $\mathbf{n}_{j}$. Here we number facets clockwise. Then we obtain a transport equation for $L_{j}(t)$ which is the length of $S_{j}(t)$ :

$$
\frac{d L_{j}(t)}{d t}=\left(\cot \psi_{j}+\cot \psi_{j+1}\right) V_{j}-\frac{1}{\sin \psi_{j}} V_{j-1}-\frac{1}{\sin \psi_{j+1}} V_{j+1}
$$

for $j=1, \ldots, r$; index $j$ is considered modulo $r$. Here $\psi_{j}=\theta_{j}-\theta_{j-1}$ (modulo $2 \pi)$ with $\boldsymbol{n}_{j}=\left(\cos \theta_{j}, \sin \theta_{j}\right)$, and $V_{j}$ denotes the normal velocity of $S_{j}(t)$ in the direction of $\boldsymbol{n}_{j}$. (2) if

We say that an essentially admissible crystal $\{S(t)\}_{t \in J}$ is a $\gamma$-regular flow of

$$
V_{j}(t)=\chi_{j} \Delta\left(\boldsymbol{n}_{j}\right) / L_{j}(t)
$$

for $j=1,2, \ldots, r$. Here $\Delta(\hat{\boldsymbol{m}})=\tilde{\gamma}^{\prime}(\hat{\theta}+0)-\tilde{\gamma}^{\prime}(\hat{\theta}-0)$ with $\hat{\boldsymbol{m}}=(\cos \hat{\theta}, \sin \hat{\theta})$ and $\tilde{\gamma}(\theta)=\gamma(\cos \theta, \sin \theta)$. We note that $\Delta(\hat{\boldsymbol{m}})$ is the length of facet of $W_{\gamma}$ with outward normal $\hat{\boldsymbol{m}}$ if $\hat{\boldsymbol{m}} \in \mathcal{N}$, otherwise $\Delta(\hat{\boldsymbol{m}})=0$. The quantity $\chi_{j}$ is called a transition number, and takes +1 (resp.-1) if the $j$-th facet is concave (resp. convex) in the direction of $\boldsymbol{n}_{j}$, otherwise $\chi_{j}=0$. We call the quantity $\Lambda_{j} \equiv \chi_{j} \Delta\left(\boldsymbol{n}_{j}\right) / L_{j}(t)$ as a nonlocal weighted curvature of the $j$-th facet with respect to $\gamma$. (We use the convention that $1 / L_{j}(t)=0$ if $L_{j}(t)=\infty$.) Thus we get a system of ODEs (4) and (5) for $L_{j}(t)$ 's. For a moment we assume that $S(0)$ is an essentially admissible closed curve.

A fundamental theory of ODE yields the (local in time) unique solvability of (4) and (5). Unless $S(t)$ shrinks to a point, self-intersects, or develops degenerate pinching at most two consecutive facets with zero nonlocal weight curvatures may disappear (i.e., the length of a facet tends to zero) at some time $T_{*}$. However, $S\left(T_{*}\right)$ remains essentially admissible, so that we can continue calculating the ODE system (5),(6) for $t>T_{*}$ starting with initial data $S\left(T_{*}\right)$ (see [11]12]). 
We say that $\{S(t)\}_{t \in J}$ is a crystalline flow with initial data $S(0)$, if there is some $t_{0}=0<t_{1}<t_{2}<\cdots<t_{l}$, such that $\{S(t)\}_{t \in J_{h}}$ is a $\gamma$-regular flow for $J_{h}=\left[t_{h}, t_{h+1}\right)$ with initial data $S\left(t_{h}\right)(h=0,1, \ldots, l-1)$, and $S(t) \rightarrow S\left(t_{h+1}\right)$ in the sense of the Hausdorff distance topology as $t \uparrow t_{h+1}$ and some facets disappear at $t_{h+1}(h=0,1, \ldots, l-2)$. By a similar argument as in [12, we see that a crystalline flow $\{S(t)\}_{t \in J}$ starting with essentially admissible closed curve $S(0)$ shrinks to a point and does not intersect nor develop degenerate pinching. A crystalline flow $\{S(t)\}_{t \in J}$ agrees with a solution by level-set approach for (2) introduced in [13], by a similar argument as in [12. The discussion in 12 is for an admissible evolving crystal but it is easy to extend to an essentially admissible evolving crystal. For convenience we recall the notion of an admissible evolving crystal. An essentially admissible crystal $S$ is called an admissible crystal if the outward unit normal vector $\boldsymbol{m}$ of each segment of $S$ belongs to $\mathcal{N}$. We say $\{S(t)\}_{t \in J}$ is an admissible evolving crystal if $S(t)$ is an admissible crystal for each $t \in J$.

\subsection{General Polygonal Initial Curve}

In the previous section we restricted an initial curve to an essentially admissible crystals. Here we shall focus on a simple, closed, polygonal initial curve $S(0)$, which is not necessarily an essentially admissible crystal. In [13, it is shown that there exists a unique level-set flow (solution) for (2) with a crystalline energy $\gamma$ starting with a general polygonal initial curve. However, it is not clear a priori whether or not the solution is described by an ODE system, since new facets whose orientation belongs to $\mathcal{N}$ are expected to be created instantaneously at the place where the property (3) is violated on $S(0)$. Moreover, it is not clear how to solve the expected ODE system since it is singular at newly created facets. In this section we give a heuristic argument to solve such a singular ODE system.

Let $\boldsymbol{m}$ and $\hat{\boldsymbol{m}}$ be the orientation of any adjacent facets $S_{j}(0)$ and $S_{j+1}(0)$ of $S(0)$. If

$$
\mathcal{M} \equiv\left\{\frac{(1-\lambda) \boldsymbol{m}+\lambda \hat{\boldsymbol{m}}}{|(1-\lambda) \boldsymbol{m}+\lambda \hat{\boldsymbol{m}}|} \in \mathcal{N} ; 0<\lambda<1\right\}
$$

is not the empty set, all facets (say, $R_{1}(t), \ldots, R_{n}(t)$, numbered clockwisely) with orientation in $\mathcal{M}$ is expected to be created between $S_{j}(0)$ and $S_{j+1}(0)$ just after $t=0$, so that the transition number of each $R_{i}(t)$ is 1 (resp. -1 ) for small $t>0$ if the bounded polygon enclosed by $S(0)$ is concave (resp. convex) near $S_{j}(0) \cap S_{j+1}(0)$. By inserting these newly created facets, our solution $S(t)$ becomes essentially admissible instantaneously. This observation should be justified by approximating $S(0)$ by essentially admissible crystals from inside and from outside with comparison principle 13 .

For a given initial polygon $S(0)$ one is able to find the place, the orientation and the transition number of the all facets that are expected to be newly created at initial time. For later convenience, we shall re-number clockwisely all facets of $S(0)$ and all facets that are expected to be created at $t=0$, i.e., the length of a newly created facet equals 0 at $t=0$. Then the expected ODE system for a 
simple, closed, polygonal initial curve $S(0)$ again becomes (41) and (5); however, the initial data $L_{j}(0)$ may be 0 . The ODE system is of the form

$$
\frac{d L_{j}(t)}{d t}=\frac{\tilde{p}_{j}}{L_{j}(t)}+\frac{\tilde{q}_{j-1}}{L_{j-1}(t)}+\frac{\tilde{r}_{j+1}}{L_{j+1}(t)}
$$

for $j=1, \ldots, r^{\prime}$; index $j$ is considered modulo $r^{\prime}$. Here numbers $\tilde{p_{j}}, \tilde{q_{j}}, \tilde{r_{j}}$ are determined uniquely by (4) and (5), since the transition number and the orientation of a newly created facet are known.

To solve the equation (6) we consider Puiseux series

$$
L_{j}(t)=\sum_{k=0}^{\infty} a_{j k} t^{k / 2},
$$

with real number $a_{j k}$. Clearly, for $j$ with $L_{j}(0)=0$ the coefficient $a_{j 0}$ must be zero. Suppose that $n$ consecutive facets, say $S_{1}(t), \ldots, S_{n}(t)$ are created at $t=0$, i.e. $L_{1}(0)=\ldots=L_{n}(0)=0$ and $L_{0}(0), L_{n+1}(0)>0$. We plug (7) into (6) and multiply $t^{1 / 2}$ with the both sides of [6]). Comparing both sides we observe that all coefficients are determined. The first coefficients $\left\{a_{j 1}\right\}_{j=1}^{n}$ have a significant meaning. If the nonlocal curvature of $S_{0}(0)$ and $S_{n+1}(0)$ equal zero, then $L_{j}(t)=a_{j 1} t^{1 / 2}$ for $j=1, \ldots, n$ exactly solves the ODE system (6) with $j=1, \ldots, n$ (as long as both $S_{0}(t)$ and $S_{n+1}(t)$ exist), since it is decoupled from the whole system (6) with $j=1, \ldots, r^{\prime}$ by the fact $\tilde{q}_{0}=0=\tilde{r}_{n+1}$. In this case the solution $\left\{a_{j 1}\right\}_{j=1}^{n}$ represents a selfsimilar expanding solution of the problem in the next section.

\subsection{Selfsimilar Expanding Solutions}

Let $\{S(t)\}_{t>0}$ be an essentially admissible evolving crystal of the form

$$
S(t)=\bigcup_{j=0}^{n+1} S_{j}(t)
$$

with nonparallel half lines $S_{0}(t)$ and $S_{n+1}(t)$. We say that $\{S(t)\}_{t>0}$ is selfsimilar if there exists an essentially admissible crystal $S_{*}$ such that

$$
S(t)=t^{1 / 2} S_{*}=\left\{t^{1 / 2} \boldsymbol{x} ; \boldsymbol{x} \in S_{*}\right\}, \quad t>0 .
$$

If $\{S(t)\}_{t>0}$ solves (6), we call $\{S(t)\}_{t>0}$ a selfsimilar expanding solution of (2). By definition $S(+0)=\lim _{t \downarrow 0} S(t)$ consists of two (nonparallel) half lines emanated from the origin. We also observe that $\cup_{j=1}^{n} S_{j}(t)$ is admissible for all $t>0$ and that the transition number of $S_{j}(t)$ is independent of $j=1, \ldots, n$ and $t>0$; it must be either -1 or +1 . It turns out that $\{S(t)\}_{t>0}$ is a selfsimilar expanding solution if and only if the length $L_{j}(t)$ of $S_{j}(t)(j=1, \ldots, n)$ solves the ODE system (6) for $t>0$ and for $j=1, \ldots, n$ with $\tilde{q}_{0}=0=\tilde{r}_{n+1}$. Note that $a_{j 1}$ of $L_{j}(t)=a_{j 1} t^{1 / 2}$ represents the length of $j$-th facet of $S_{*}$ for $j=1, \ldots, n$. 
Theorem For a given oriented closed cone $C$ (with connected interior) there exists a unique selfsimilar expanding solution $S(t)$ such that $S(+0)$ agrees with the boundary of $C$ (see [14]).

From ODE system (6) we see that this problem is equivalent to the unique solvability of algebraic equation

$$
\left[\begin{array}{l}
a_{n} \\
a_{n-1} \\
a_{n-2} \\
\vdots \\
a_{2} \\
a_{1}
\end{array}\right]=2\left[\begin{array}{cccccc}
\tilde{p}_{n} & \tilde{q}_{n-1} & & & \\
\tilde{r}_{n} & \tilde{p}_{n-1} & \tilde{q}_{n-2} & & 0 \\
& \tilde{r}_{n-1} & \tilde{p}_{n-2} & \tilde{q}_{n-3} & \\
& & \ddots & \ddots & \ddots & \\
& & & \tilde{r}_{3} & \tilde{p}_{2} & \tilde{q}_{1} \\
& 0 & & & \tilde{r}_{2} & \tilde{p}_{1}
\end{array}\right]\left[\begin{array}{l}
1 / a_{n} \\
1 / a_{n-1} \\
1 / a_{n-2} \\
\vdots \\
1 / a_{2} \\
1 / a_{1}
\end{array}\right]
$$

for $a_{j}=a_{j 1}(j=1,2, \ldots, n)$. We solved this equation by a method of continuity while we proved the uniqueness of a solution by a geometric observation 14.

\section{Numerical Method for Obtaining a Crystalline Flow}

In this section, we describe a numerical method for obtaining a crystalline flow starting from a given polygon that is not necessarily an essentially admissible crystal. For each adjacent facets with orientation $\boldsymbol{m}$ and $\hat{\boldsymbol{m}}$ of the initial polygon, if $\mathcal{M} \neq \emptyset$ then all facets with orientation in $\mathcal{M}$ should newly sprout out instantaneously, so that the given polygon becomes essentially admissible instantaneously. Once the polygon becomes essentially admissible, no new facet sprout out and remains essentially admissible through the evolving process.

We calculate singular ODE system (4) and (5) by the Euler method. A special treatment is necessary to get approximate length of newly created facets at first time step $\Delta t$. We take $L_{j}(\Delta t)=a_{j} \sqrt{\Delta t}$ for such facets, where $a_{j}$ is a numerical solution of (8). To solve (8) numerically, as in [14] we rewrite (8) with $\alpha_{j}=1 / a_{j}$ :

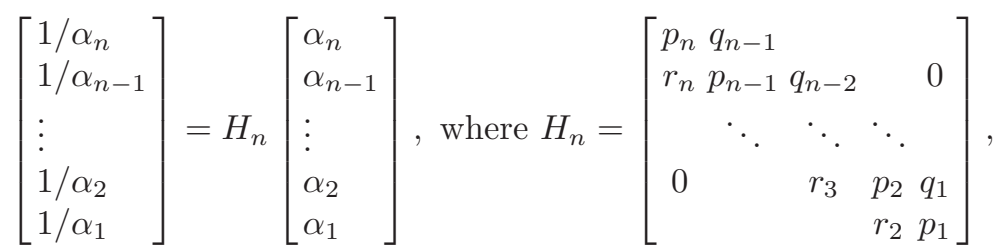

$p_{j}=2 \tilde{p}_{j}, q_{j}=2 \tilde{q}_{j}$, and $r_{j}=2 \tilde{r}_{j}$. We introduce extra parameter $s \in[0,1]$ by replacing $H_{n}$ by $K_{n}(s)$ in (9).

$$
K_{n}(s)=\left[\begin{array}{ccccc}
p_{n} & s q_{n-1} & & & \\
s r_{n} & p_{n-1} & s q_{n-2} & & 0 \\
& \ddots & \ddots & \ddots & \\
0 & & s r_{3} & p_{2} & s q_{1} \\
& & & s r_{2} & p_{1}
\end{array}\right]
$$


Evidently $\left[1 / \alpha_{j}(0)\right]=K_{n}(0)\left[\alpha_{j}(0)\right]$ can be easily solved: $\alpha_{j}(0)=1 / \sqrt{p_{j}}$. Based on [14], we calculate the numerical solution of (8) as follows.

1. Set $\alpha_{j}=1 / \sqrt{p_{j}}=2\left[\cot \phi_{j}+\cot \phi_{j+1}\right] \chi_{j} \Delta\left(\boldsymbol{n}_{j}\right)$ for initial values.

2. Apply the Newton-Rapson method to obtain the numerical solution of $\alpha_{j}$.

3. Calculate $a_{j}=1 / \alpha_{j}$, and set $L_{j}(\Delta t)=a_{j} \sqrt{\Delta t}$, where $\Delta t$ is the time interval.

Once new facets are inserted into a given polygon, then the length of each facet $L_{j}(i \Delta t)$ is calculated at each time step by the system of (4) and (5). Note that $L_{j}(t)=a_{j} t^{1 / 2}$ is the exact solution if the speeds of both facets bounding newly created facets are zero.

\section{Experimental Results}

In the first experiment, we used a regular 16-polygon as the Wulff shape, and a square as an initial contour. Let $\boldsymbol{m}_{i}(i=1,2, \cdots, 16)$ denote the outward unit normals of the Wulff shape. We set that the arg $\boldsymbol{m}_{i}=\pi-\pi(i-1) / 8$ (the facet number is counted clockwise). Let $F_{j}$ denote the $j$ th facet of the initial contour, and $\boldsymbol{n}_{j}(j=1,2,3,4)$ be the outward unit normal of $F_{j}$. Assume that arg $\boldsymbol{n}_{j}=\pi-\pi(j-1) / 2$. Then, three new facets sprout out at each corner of the square. For example, between $F_{1}\left(\arg \boldsymbol{n}_{1}=\pi\right)$ and $F_{2}\left(\arg \boldsymbol{n}_{2}=\pi / 2\right)$ of the given square, three facets sprout out of which normals are parallel to $\boldsymbol{m}_{2}, \boldsymbol{m}_{3}$, and $\boldsymbol{m}_{4}$, respectively.

In order to obtain the quantities of $a_{j}$, we solve next equations that correspond to (8).

$$
\left[\begin{array}{l}
a_{3} \\
a_{2} \\
a_{1}
\end{array}\right]=\left[\begin{array}{lll}
p & q & 0 \\
q & p & q \\
0 & q & p
\end{array}\right]\left[\begin{array}{l}
1 / a_{3} \\
1 / a_{2} \\
1 / a_{1}
\end{array}\right] \text {, where } p=\frac{4}{\tan (\pi / 8)} \text { and } q=-\frac{2}{\tan (\pi / 8)} .
$$

Let $\alpha=1 / a_{1}=1 / a_{3}$ and $\beta=1 / a_{2}$. The equation(11) can be solved analytically:

$$
\left\{\begin{array}{l}
\alpha=-1 / 2 q(p \beta-1 / \beta), \\
\beta=\left[\left(p^{2}+q^{2}\right)+\sqrt{\left(p^{2}+q^{2}\right)^{2}-p^{2}\left(p^{2}-2 q^{2}\right)}\right] /\left[p\left(p^{2}-2 q^{2}\right)\right] .
\end{array}\right.
$$

We can calculate the quantities of $a_{j}$ s using $a_{1}=a_{3}=1 / \alpha$ and $a_{2}=1 / \beta$. The values $p$ and $q$ in (12) are known as shown in (11). The values are $a_{1}=a_{3} \simeq 1.68$ and $a_{2} \simeq 1.29$, respectively. Three facets sprout out with symmetric shape in this case. It should be noted that the shape of the set of new facets are not same with the shape of the corresponding part of the Wulff shape. In this case, the middle facet is shorter than the neighbors, in spite that the Wulff shape is regular.

Figure 2 shows some experimental results of crystalline flow. The initial contour is common to all, but the Wulff shape is different. As described before, the Wulff shape plays the role of a unit circle for a classical curve shortening flow. Because the proposed method can obtain a crystalline flow from a non essentially admissible crystal, any simple and convex polygon can be used for the Wulff shape. 


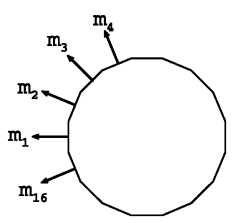

Wulff shape

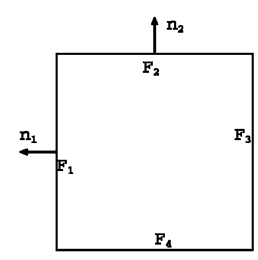

Initial Contour

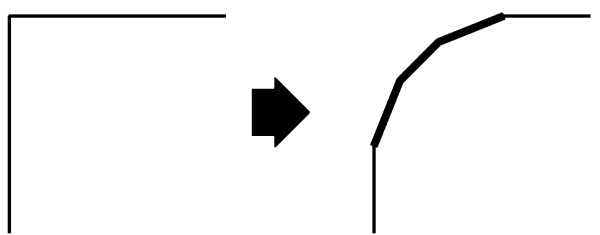

Facet Insertion

Fig. 1. An example of the the Wulff shape and an initial contour. An analytic solution can be calculated in this case. Three new facets are inserted at the beginning as shown in the this figure. It should be noted that the middle facet is shorter than side ones.

(A)
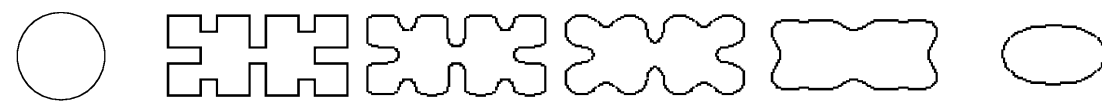

(B)
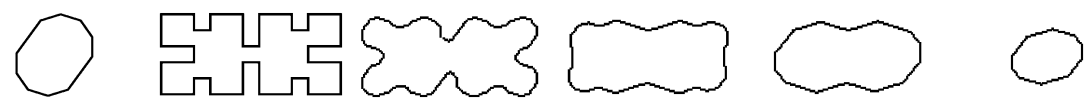

(C)
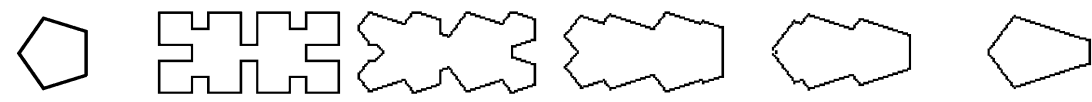

(D)
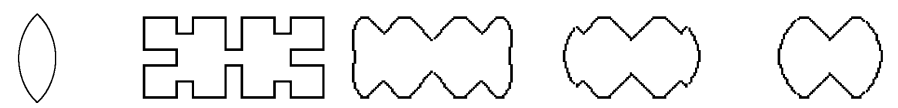

0

Fig. 2. Examples of the crystalline flow. The initial contour is common to all, and is shown in the second column. The Wulff shapes are shown at the left: (A) a regular 30-polygon, (B) a decagon two of which facets are longer than others, (C) a regular pentagon, and (D) a 30-polygon each of which facet has the same length.

\section{Conclusion}

A numerical method for obtaining a crystalline flow from a given polygon that is not essentially admissible is presented. The method enables to use any simple and convex polygon for the Wulff shape, because a crystalline flow can be obtained from any simple polygon even if it is not essentially admissible.

In many cases, a contour in an image is given as a polygon. For example, a contour represented with a chain-code is a polygon that consists of short facets. Because the nonlocal curvature $\Lambda_{\gamma}$ is determined by the facet length, no approximation is needed for the calculation of the curvature. In addition, because each facet moves with keeping its direction, it is not difficult to trace every facet through the evolving process. We believe that those features of a crystalline flow are useful for multi-scale contour figure analysis. 


\section{References}

1. Koenderink, J. J.: The Structure of Images, Biological Cybernetics, 50 (1984) 363370

2. Alvarez, L. and Guichard, F.: Axioms and Fundamental Equations of Image Processing, Arch. Rational Mech. Anal., 123 (1993) 199-257

3. Mokhtarian, F. and Mackworth, A.: A Theory of Multiscale, Curvature-Based Shape Representation for Planner Curves, IEEE Transactions on Pattern Analysis and Machine Intelligence, 14, No.8 (1992) 789-805

4. Rattarangsi, A. and Chin, R. T.: Scale-Based Detection of Corners of Planar Curves, IEEE Transactions on Pattern Analysis and Machine Intelligence, 14, No.4 (1992) 430-449

5. Kimia, B. B., and Tannenbaum, A. R. and Zucker S. W.: Shapes, Shocks, and Deformations I: The Components of Two-Dimensional Shape and the ReactionDiffusion Space, International Journal of Computer Vision, 15 (1995) 189-224

6. Hontani, H. and Deguchi, K.: Multi-Scale Image Analysis for Detection of Characteristic Component Figure Shapes and Sizes, Proceedings of 14th International Conference on Pattern Recognition (1998) 1470-1472

7. Osher, S. and Sethian, J. A.: Fronts propagating with curvature-dependent speed: Algorithms based on Hamilton-Jacobi formulations, J. Comput. Phys. 79 (1988) $12-49$

8. Evans, L. C. and Spruck, J.: Motion of level-sets by mean curvature, I, J. Differential Geometry, 33 (1991) 635-681

9. Chen, Y.-G., Giga, Y. and Goto, S.: Remarks on viscosity solutions for evolution equations, J. Differential Geometry, 33 (1991) 749-786

10. Angenent, S. B. and Gurtin, M. E.: Multiphase thermomechanics with interfacial structure 2. Evolution of an isothermal interface, Arch. Rational Mech. Anal., 108 (1989) 323-391

11. Taylor, J.: Constructions and conjectures in crystalline nondifferential geometry, Proceedings of the Conference on Differential Geometry, 52, Pitman, London (1991) 321-336

12. Giga, M. -H. and Giga, Y.: Crystalline and level-set flow - Convergence of a crystalline algorithm for a general anisotropic curvature flow in the plane, Free boundary problems: theory and applications I (ed. N. Kenmochi) Gakuto International Ser. Math. Sci. Appl., 13 (2000) 64-79

13. Giga, M.-H. and Giga, Y.: Generalized Motion by Nonlocal Curvature in the Plane, Arch. Rational Mech. Anal., 159 (2001) 295-333

14. Giga, M. -H., Giga, Y. and Hontani, H.: Selfsimilar solutions in motion of curves by crystalline energy, Minisymposium lecture of 5th International Congress on Industrial and Applied Mathematics, Sydney, (2003), July

15. Gurtin, M. E.: Thermomechanics of Evolving Phase Boundaries in the Plane, Oxford, Clarendon Press (1993)

16. Giga, Y.: A level set method for surface evolution equations, Sugaku 47 (1993) 321-340: Eng. translation, Sugaku Exposition 10 (1995), 217-241

17. Giga, Y. and Goto, S.: Motion of hypersurfaces and geometric equations, J. Mathematical Society Japan, 44 (1992) 99-111 